\title{
Empowerment and Problems in Health Care Access among Currently Married Women in Myanmar: A Secondary Data Analysis on MDHS 2015-16
}

\section{CURRENT STATUS: POSTED}

Research Square

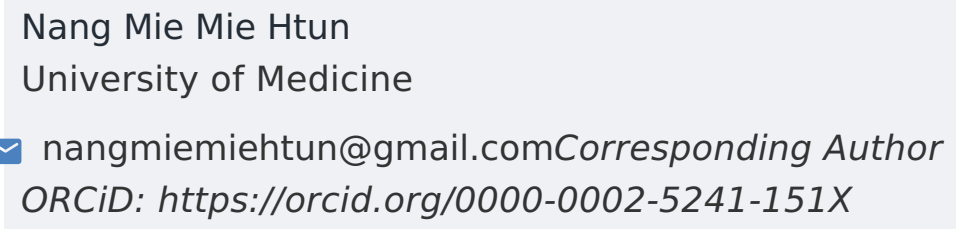

\section{Zar Lwin Hnin}

Department of Preventive and Social Medicine,University of Medicine,Mandalay, Ministry of Health and Sports, Myanmar

Win Khaing

Department of Preventive and Social Medicine,University of Medicine,Mandalay, Ministry of Health and Sports,Myanmar

\section{DOI:}

$10.21203 / \mathrm{rs} .2 .23098 / \mathrm{v} 1$

\section{SUBJECT AREAS}

Health Policy

\section{KEYWORDS}

women's empowerment, problems in accessing health care, Demographic and Health Surveys, Myanmar, knowledge, decision power, beating, labour force 
Abstract

Background: Women's health outcomes are influenced by the lack of access to health care and their inability to make decisions for themselves. This study was conducted to identify the association between women's empowerment and the problems in assessing health care among currently married women aged 15-49 years.

Method: A secondary analysis by using Myanmar Demographic and Health Survey (MDHS) (2015-16) data, which included all 15 regions of Myanmar. In the study, $(7,759)$ eligible currently married women aged 15-49 years were included.

Result: Among eligible women, 52.43\% (95\% Cl: 0.51-0.53) had problems in accessing health care. Women with medium and high empowerment scores were less likely to experience problems in accessing health care compared to women who got low score $(\mathrm{aOR}=0.85,95 \% \mathrm{Cl}: 0.73-0.98)$ $(\mathrm{aOR}=0.55,95 \% \mathrm{Cl}: 0.47-0.65)$ respectively. Women from rural area (aOR=1.41, 95\% $\mathrm{Cl}: 1.15-1.72)$ and women living in Chin State, one of the least developed states, (aOR=1.84, 95\% Cl: 1.38-2.46) had faced more problems in accessing health care, on the other hand, the problems were seen to be reduced in the case of women aged over 35 years $(\mathrm{aOR}=0.66,95 \% \mathrm{Cl}: 0.47-0.94)$, and those who had an educated husband ( $\mathrm{aOR}=0.76,95 \% \mathrm{Cl}: 0.66-0.86)$, a husband with a white collar job $(\mathrm{aOR}=0.71,95 \% \mathrm{Cl}: 0.56-0.89)$, and those living with an extended family $(\mathrm{aOR}=0.74,95 \% \mathrm{Cl}: 0.66-$ $0.84)$.

Conclusion: The study showed when the women are more empowered, they might have less problems in accessing health care. These finding would contribute to the policy formulation in reducing health inequity issues in terms of increasing women's empowerment by enabling women getting equal right to education and jobs.

Key words: women's empowerment, problems in accessing health care, Demographic and Health Surveys, Myanmar, knowledge, decision power, beating, labour force

\section{Full Text}

Due to technical limitations, full-text HTML conversion of this manuscript could not be completed. However, the manuscript can be downloaded and accessed as a PDF.

Figures 


\section{Women's Empowerment}

\section{Labor force participation}

$\checkmark$ Work all years, Work at the last 12 months, Women's occupation, Earn more than husband, Types of payment women get, For whom women work

\section{Disagreement to justification toward wife} beating

$\checkmark$ Burn food, Argue, Neglect children, Refuse sex, Going out without husband's permission

\section{Decision making power}

$\checkmark$ Visiting family, Own health care, Husband's earning, Household purchase

\section{Women's knowledge level}

Education level, Access to media

Outcome: Access to Health Care

Getting medical help for self:

$\checkmark$ Getting permission to go

$\checkmark$ Getting money needed for treatment

$\checkmark$ Not wanting to go alone

\section{Covariates}

$\checkmark$ Women's age

$\checkmark$ Husband's age, education, occupation

$\checkmark$ Inter-marital age difference

$\checkmark$ Total number of living children

$\checkmark$ Family structure

$\checkmark$ Wealth quintile

$\checkmark$ Residence (Urban/ rural)

$\checkmark$ States and Regions

Figure 1

Conceptual framework 


\section{Women's Empowerment}

\section{Labor force participation}

$\checkmark$ Work allyears, Work at the last 12 months, Women's occupation, Earn more than husband, Types of payment women get, For whom women work

\section{Disagreement to justification toward wife beating}

$\checkmark$ Burn food, Argue, Neglect children, Refuse sex, Going out without husband's permission

\section{Decision making power}

$\checkmark$ Visiting family, Own health care, Husband's earning, Household purchase

\section{Women's knowledge level}

$\checkmark$ Education level, Access to media

\section{Covariates}

$\checkmark$ Women's age

$\checkmark$ Husband's age, education, occupation

$\checkmark$ Inter-marital age difference

$\checkmark$ Total number of living children

$\checkmark$ Family structure

$\checkmark$ Wealth quintile

$\checkmark$ Residence (Urban/ rural)

$\checkmark$ States and Regions

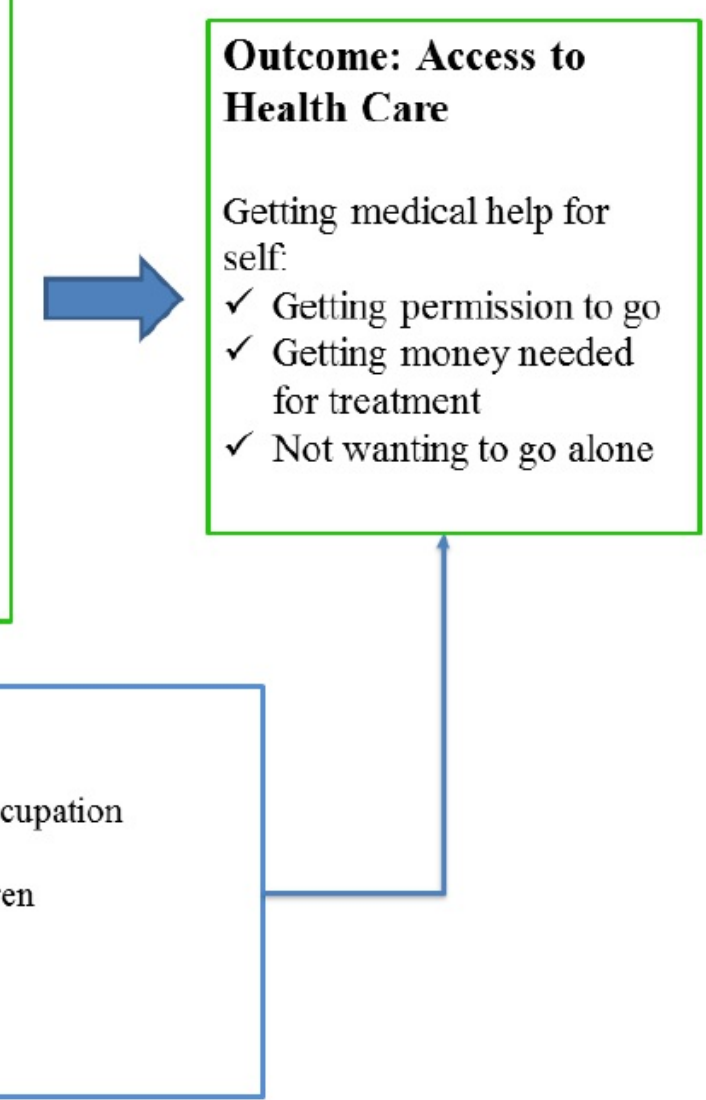

Figure 1

Conceptual framework 


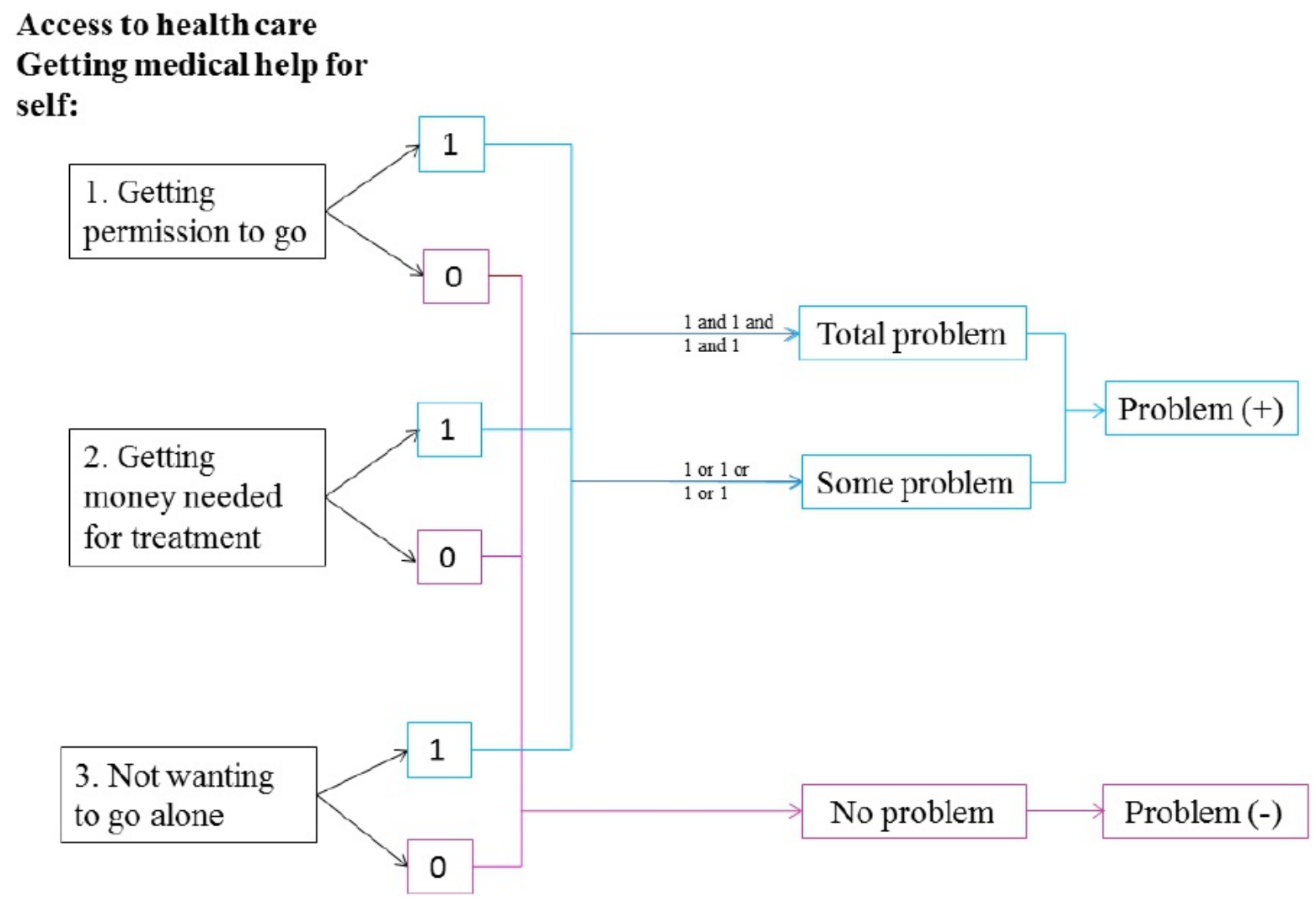

Figure 2

Scoring system for access to health care 


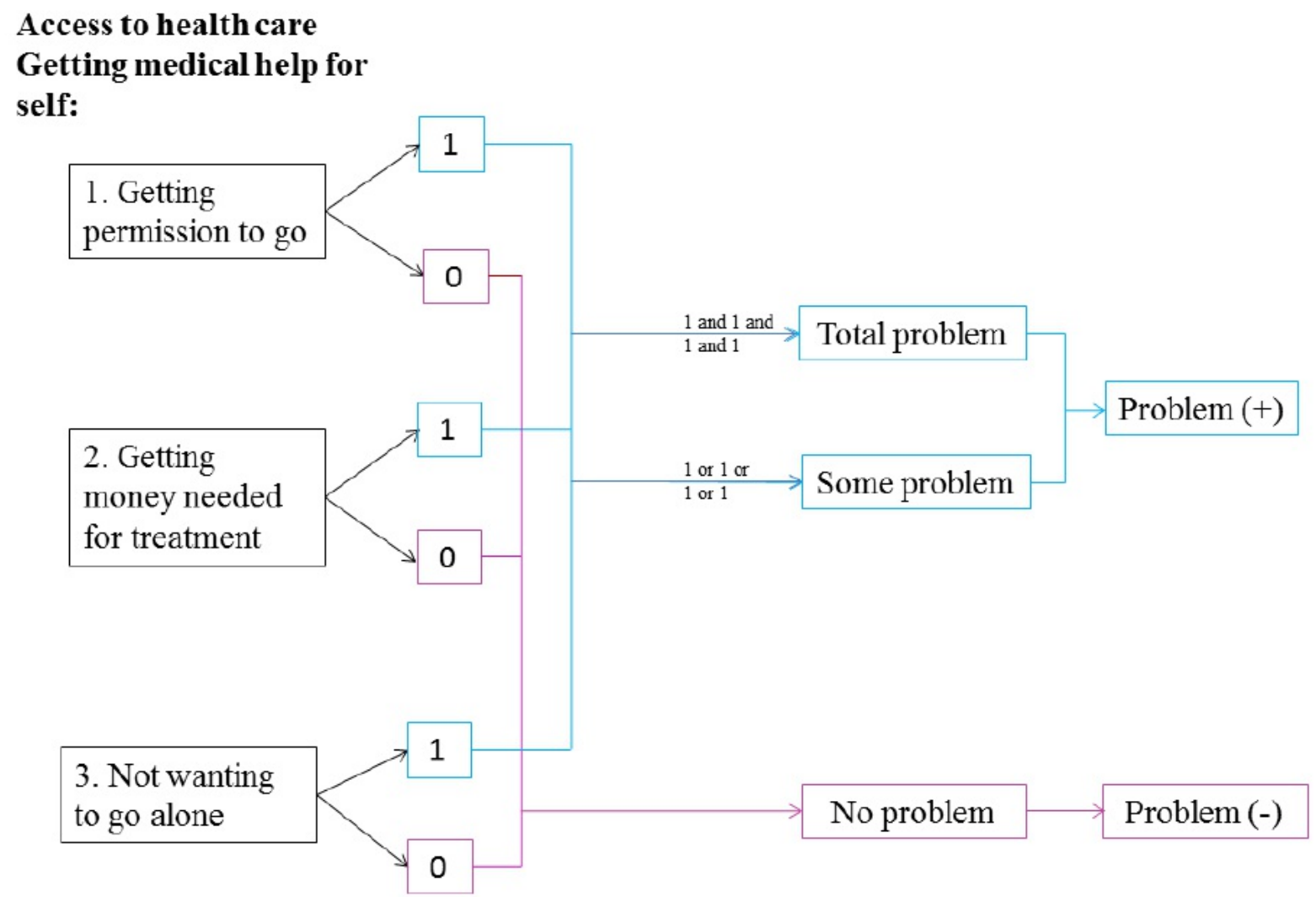

Figure 2

Scoring system for access to health care 


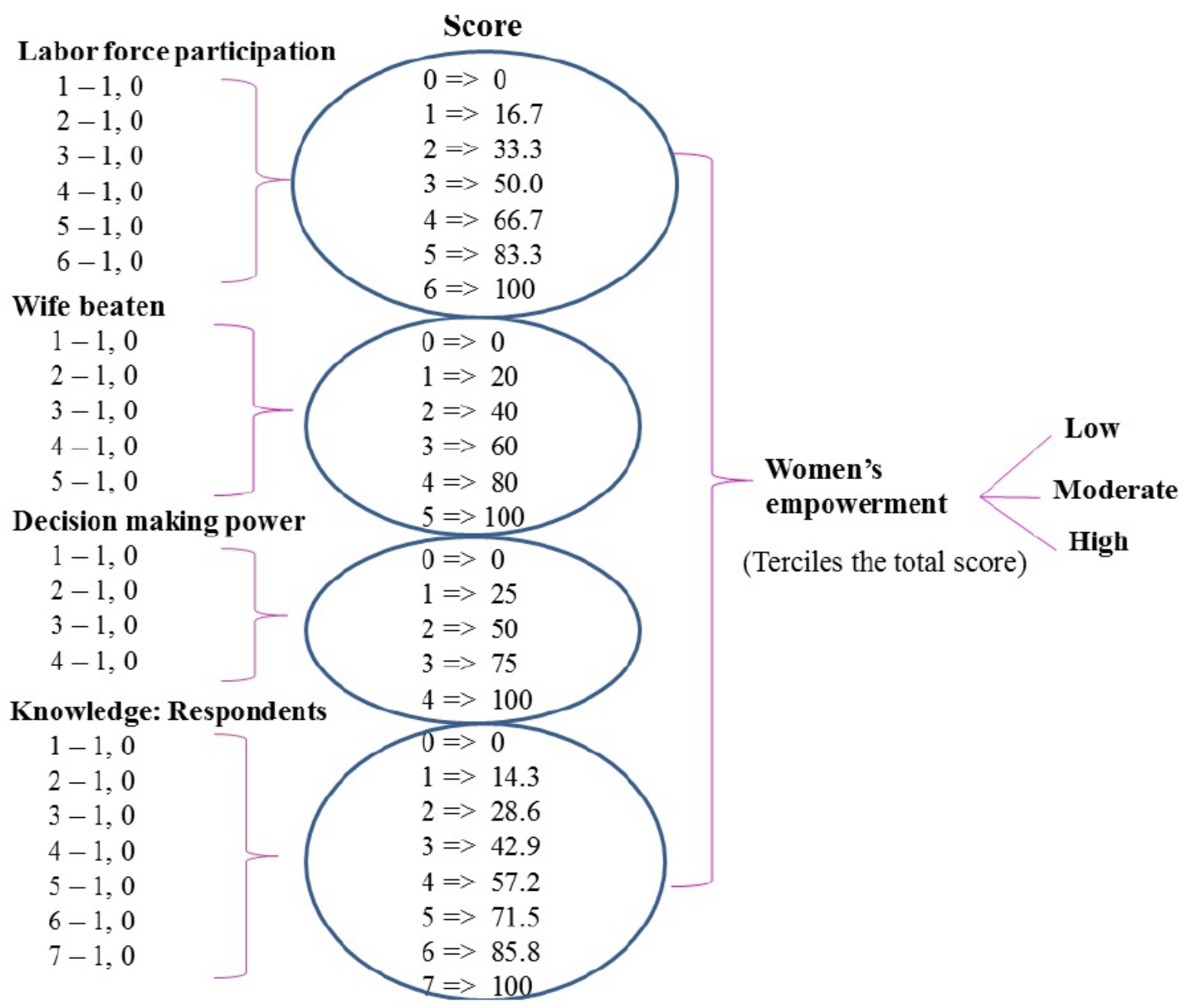

Figure 3

Scoring system for domains of women's empowerment 


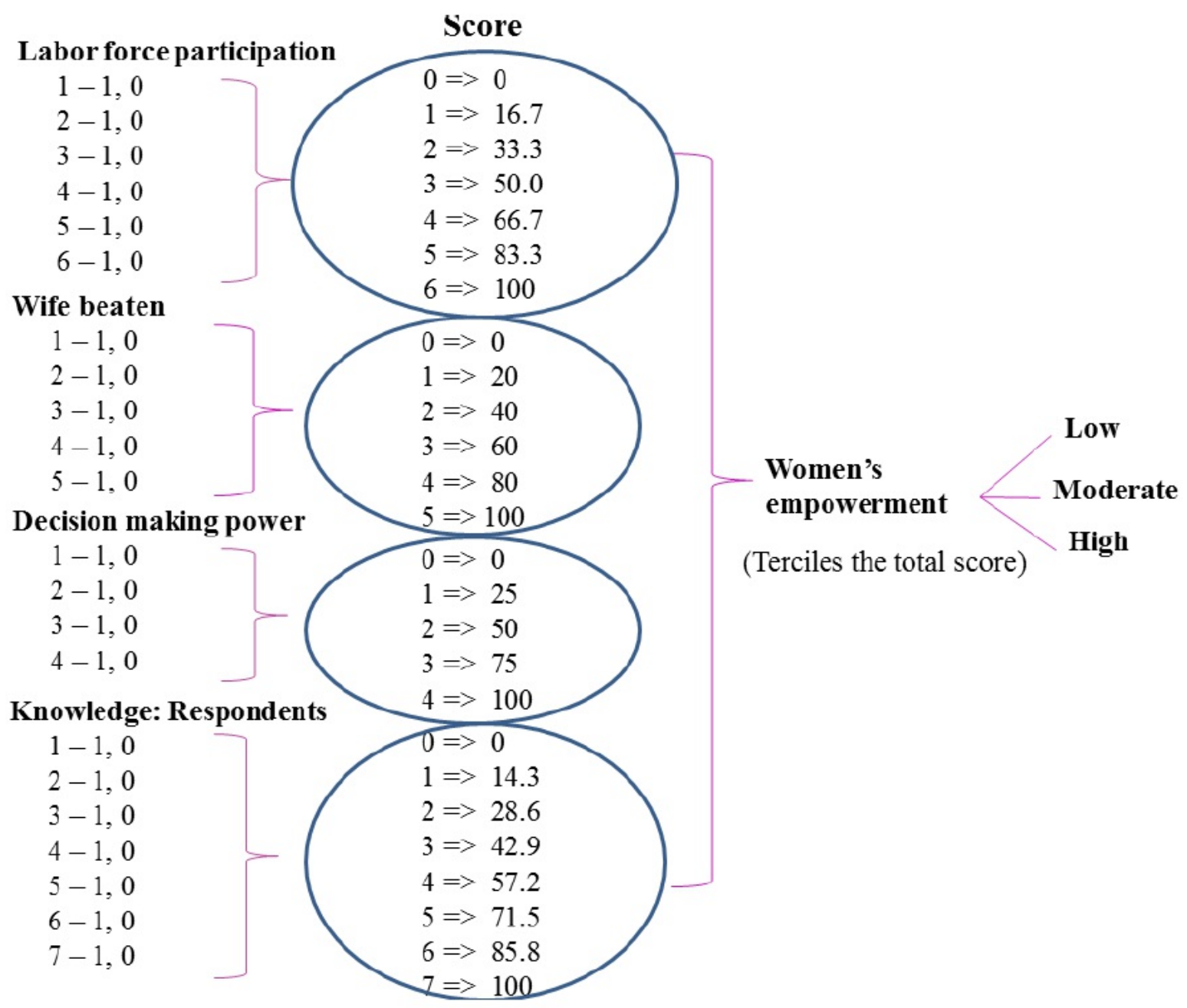

Figure 3

Scoring system for domains of women's empowerment 